'Grupo de Investigación

Promoción y Prevención

Farmacéutica, Departamento

de Farmacia, Universidad de

Antioquia, Medellín, Colombia.

${ }^{2}$ Grupo Investigación Clínica

HPTU. Hospital Pablo Tobón

Uribe, Medellín, Colombia.

${ }^{a}$ Químico-Farmacéutico.

${ }^{\mathrm{b}} \mathrm{Msc}$.

CPhD.

Apoyo financiero: El grupo

Promoción y Prevención

Farmacéutica recibió financiación de la convocatoria de

sostenibilidad 2018-2019 del

Comité para el Desarrollo de la Investigación de la Universidad de Antioquia.

Los autores declaran no tener ningún conflicto de Interés.

Recibido el 1 de agosto de 2017, aceptado el 14 de marzo de

2018.

Correspondencia a: Elkyn Johan Granados Vega Medellín Colombia elkyn.granados@udea.edu.co

\section{Disglucemia asociada a fluoroquinolonas: una revisión estructurada}

\author{
JOHAN GRANADOS 1 1,2,a,b, MAURICIO CEBALLOS ${ }^{1, \mathrm{a}, \mathrm{b}}$, \\ PEDRO AMARILES ${ }^{1, \mathrm{a}, \mathrm{c}}$

\section{Hypo or hyperglycemia associated with fluoroquinolone use}

Fluoroquinolone type antimicrobials can cause hypo or hyperglycemia in certain patients. We performed a structured review about this side effect, searching articles published in English or Spanish with full text access in PubMed/ Medline. The following MESH terms were used: Hypoglycemia, Hyperglycemia, Quinolones, Ciprofloxacin, Levofloxacin, Moxifloxacin. Additionally, we evaluated the clinical relevance of potential drug interactions, based on the probability of occurrence and the severity of the interaction effect. We obtained 42 publications about the issue; 22 references were selected, where the severity of the interaction in patients with risk factors was evaluated. Patients receiving antidiabetic medications and with risk factors such as advanced age and renal failure may be more likely to have a severe hypoglycemia. In these patients, this drug interaction should be considered clinically relevant since its risk is high or very high.

(Rev Med Chile 2018; 146: 618-626)

Key words: Hyperglycemia; Hypoglycemia; Quinolones.

\section{E} $\mathrm{n}$ la prevención de la aparición y la progresión de complicaciones crónicas asociadas a la diabetes mellitus y su tratamiento, resulta clave alcanzar y mantener valores de normoglucemia (prepandial: $80-130 \mathrm{mg} / \mathrm{dL}$ y postprandial menor $180 \mathrm{mg} / \mathrm{dL}$ ) y de hemoglobina glicosilada A1 inferiores a $7 \%^{1}$. El logro de dicho objetivo en ocasiones se ve afectado por estados de hipoglucemia, atribuidos a las propiedades farmacológicas y mecanismo de acción de los fármacos antidiabéticos, los cuales pueden ser graves y poner en riesgo la vida de los pacientes ${ }^{2}$; adicionalmente la respuesta de los pacientes al tratamiento con medicamentos antidiabéticos puede verse afectada por interacciones medicamentosas ${ }^{3}$. La medicación concomitante puede causar interacciones farmacocinéticas (cambio en el efecto debido a fluctuaciones en los niveles plasmáticos de los fármacos antidiabéticos) o farmacodinámicas (cambio en el efecto sin variaciones en las concentraciones del antidiabético), generando con ello, estados de hipoglucemia o de hiperglucemia ${ }^{3,4}$, favoreciendo la liberación de insulina, disminuyendo la resistencia periférica a la insulina o reduciendo la producción hepática de glucosa.

Las quinolonas son una clase de antibacterianos ampliamente utilizados para tratar una gran variedad de infecciones bacterianas en humanos. Son antimicrobianos que inhiben el resellado de la ruptura de doble cadena del $\mathrm{ADN}$ generado por la ADN girasa y ADN topoisomerasa IV en el proceso de replicación de la información genética ${ }^{5}$.

Estos medicamentos pueden producir disglucemias ${ }^{3}$, consideración que debería tenerse presente en pacientes que se encuentran en terapia con antidiabéticos. Korbel y Spencer describieron que hasta $20 \%$ de los reingresos hospitalarios en pacientes diabéticos se asociaron a infecciones del 
tracto urinario, piel, neumonía y sepsis, susceptibles a ser tratados con quinolonas ${ }^{6}$.

Algunos autores, utilizando modelos animales han presentado explicaciones del posible mecanismo de las hipoglucemias asociadas al uso de fluoroquinolonas. En este sentido, Ghaly $\mathrm{H}$ et al., evidenciaron que la ciprofloxacina y la moxifloxacina generan un aumento de la liberación de insulina, bloqueando los canales de K+ ATP-sensibles. Además, reportaron que el efecto hipoglucemiante es dependiente de la dosis (Figura 3 ) y que es mayor con moxifloxacina que con ciprofloxacina ${ }^{7}$. Por su parte, Saraya et al., identificaron que las fluoroquinolonas causarían un aumento en la secreción de insulina, de forma similar a como lo hacen las sulfonilureas ${ }^{8}$.

El mecanismo que produce una respuesta hiperglucémica con el uso de quinolonas aún no es claro, aunque existen datos de estudios en animales que presentaron una disminución en los gránulos secretores en las células pancreáticas $\beta$ cuando se expusieron a gatifloxacina, lo que a su vez pudo conducir a una disminución en los niveles de insulina sérica y provocar hiperglucemia ${ }^{9}$.

A la fecha no se dispone de una revisión estructurada que detalle la frecuencia y características asociadas, incluyendo las diferencias en el efecto de grupo, de las disglucemias asociadas a las fluoroquinolonas, con el soporte de los estudios analíticos y reportes de caso disponibles. Por tanto, el objetivo de este trabajo fue identificar y caracterizar la incidencia del efecto hipoglucemiante e hiperglucemiante asociado a las fluoroquinolonas en pacientes con diabetes y sin diabetes.

\section{Materiales y Métodos}

Se realizó una revisión estructurada (Revisión que tiene una estrategia de búsqueda definida que permite ser replicable) en PubMed/Medline sobre el efecto hipoglucemiante e hiperglucemiante de las fluoroquinolonas en humanos, se buscaron artículos publicados en inglés o español, con acceso a texto completo entre septiembre de 2006 y septiembre de 2017. La revisión se complementó con la inclusión de las referencias bibliográficas de los artículos consideradas de interés para el tema. La búsqueda se realizó con los siguientes términos MESH: Hypoglycemia, Hyperglycemia, Quinolones, Ciprofloxacin, Levofloxacin,
Moxifloxacin (Anexo 1). Los criterios de inclusión fueron: 1) describir en el título o resumen información del cambio en la glucemia asociada al tratamiento con fluoroquinolonas; y 2) disponibilidad de acceso al texto completo. Por su parte, los criterios de exclusión fueron: 1) artículos sin ninguna relación con los objetivos de la revisión; 2) artículos con información limitada para evaluar relevancia clínica de la interacción o sin desenlace clínico o evaluación de la causalidad en reportes de caso; y 3) artículos relacionados con gatifloxacina (retirada del mercado en el 2009).

Los artículos identificados fueron leídos y seleccionados por 3 investigadores de forma independiente. Para ello, se revisaron los títulos y resúmenes de todas las publicaciones identificadas para decidir su elegibilidad. Posteriormente, los artículos seleccionados se analizaron conjuntamente y, por consenso, se definió su inclusión o exclusión. Para la evaluación de la inclusión de las cohortes se tomó como base la guía la declaración STROBE de lectura de estudios observacionales.

La clasificación de la relevancia clínica de las interacciones medicamentosas se estableció con base en la gravedad y probabilidad de ocurrencia de la misma ${ }^{10}$.

\section{Gravedad de la interacción ${ }^{10}$}

Grave. La interacción puede causar daño o lesión al paciente. La consecuencia del resultado clínico negativo de la farmacoterapia puede causar o generar en el paciente la muerte, riesgo para la vida, hospitalización, una incapacidad permanente o significativa, anomalías congénitas o malformaciones al nacer, al igual que otros efectos que a juicio médico puedan comprometer la integridad del paciente y generar la necesidad de realizar una intervención quirúrgica para evitar la muerte, hospitalización o anomalías congénitas.

Moderada. La interacción genera la necesidad de realizar un seguimiento del paciente. La consecuencia del resultado clínico negativo de la farmacoterapia puede causar una modificación (cambio o interrupción) de la farmacoterapia o el empleo de nuevos fármacos para tratar el problema relacionado con medicamentos, o bien la prolongación de la hospitalización del paciente.

Leve. La interacción no causa daño al paciente. La consecuencia del resultado negativo de la medicación no requiere la modificación (cambio 
o interrupción) de la farmacoterapia o el empleo de nuevos fármacos para tratar el problema relacionado con medicamentos ni prolonga la hospitalización del paciente.

\section{Probabilidad de aparición de la interacción ${ }^{10}$}

Por su parte, para la probabilidad de aparición de la interacción se establecieron 3 categorías: definida, probable o posible a partir del tipo de estudio que ha documentado la interacción y que ha sido publicado en revistas con revisión previa e indexada en bases de datos:

Definida: Interacción documentada en metaanálisis, revisiones sistemáticas o ensayos clínicos aleatorizados o no aleatorizados.

Probable: Interacción documentada en estudios analíticos o por la descripción de 3 o más casos.

Posible: Interacción documentada por la descripción de menos de 3 casos o por recomendaciones de grupos de expertos.

A partir de las combinaciones posibles de gravedad y probabilidad de aparición, las interacciones se agruparon en 4 categorías:

Nivel 1: Riesgo muy alto (resultante de la combinación grave y definida, o grave y probable. En este caso el uso simultáneo de los medicamentos se consideró absolutamente contraindicado).

Nivel 2: Riesgo alto (resultante de la combinación grave y posible, moderada y definida o moderada y probable. El uso simultáneo requiere ajustes en la dosificación y valoración clínica permanente de forma cuantitativa de la efectividad y seguridad de la combinación de los fármacos interactuantes).

Nivel 3: Riesgo Medio (resultante de la combinación moderada y posible, leve y definida o leve y probable. También es necesario un monitoreo clínico frecuente y un análisis sobre la necesidad de ajustar la dosis).

Nivel 4: Riesgo Bajo (resultante de la combinación leve y posible. La interacción es de escasa relevancia clínica).

\section{Análisis estadístico}

Se realizaron análisis estadísticos descriptivos, en el caso de los reportes de caso, se calcularon medias y proporciones. Adicionalmente, se realizó un forestplot, gráfica utilizada para resumir visualmente los riesgos relativos (RR) reportados en todos estudios de cohorte. Es importante destacar que con este análisis no se pretendió establecer un valor de la combinación de los estudios (meta-análisis) ya que la intención del estudio es descriptiva, por tanto, no se presentó el rombo para el RR.

\section{Resultados}

En total, 42 referencias bibliográficas fueron identificadas y se seleccionaron 24 artículos, en la Figura 1 se ilustra el proceso de identificación y selección de los manuscritos. Adicionalmente, se complementó la búsqueda con 2 artículos de interés, identificados en las referencias de los trabajos seleccionados. En dichos artículos se identificaron 6 interacciones, 3 valoradas de riesgo muy alto (nivel 1), para generar hipoglucemia, resultante de la combinación grave/probable, y las 3 restantes de riesgo medio (nivel 3) de causar hiperglucemia, producto de la combinación leve/probable, tal como se detalla en la Tabla 1. Los 24 artículos revisados mostraron que las fluoroquinolonas pueden causar disglucemias, siendo la hipoglucemia la alteración de mayor relevancia debido a que genera riesgo para la vida de los pacientes.

Acorde con los 15 reportes de caso, la edad media (DE) de los pacientes con hipoglucemia fue de $66,1(12,7)$ años, el 53\% hombres, 66\% tenía diagnóstico de diabetes, el $13 \%$ no tenía de diabetes y de $20 \%$ no se reportó el dato del diagnóstico. El $60 \%$ de los pacientes presentaron alteración renal, mientras que $13 \%$ alteración hepática. En el caso de los 10 pacientes con diabetes, $60 \%$ tenía prescrito una sulfonilurea. En los 15 reportes de caso, la levofloxacina $(73,3 \%)$, seguido de la moxifloxacina $(20,0 \%)$ y finalmente la ciprofloxacina $(6,6 \%)$ fueron las fluoroquinolonas más asociadas a eventos hipoglucémicos. En todos los reportes de caso la gravedad de la hipoglucemia fue grave ya que fue causa de ingreso a hospitalización. La edad avanzada, la falla renal y la utilización concomitante de sulfonil-ureas podrían considerarse como factores de riesgo para hipoglucemia. Los resultados se detallan en la Tabla 2.

En pacientes con diabetes, en orden decreciente, las fluoroquinolonas con mayor riesgo de hipoglucemias fueron levofloxacina $>$ moxifloxacina > ciprofloxacina. Por su parte, sin diferenciar por la presencia de diabetes (pacientes con o sin diabetes -población general-) el riesgo fue mayor con moxifloxacina seguida de ciprofloxacina y finalmente levofloxacina. 


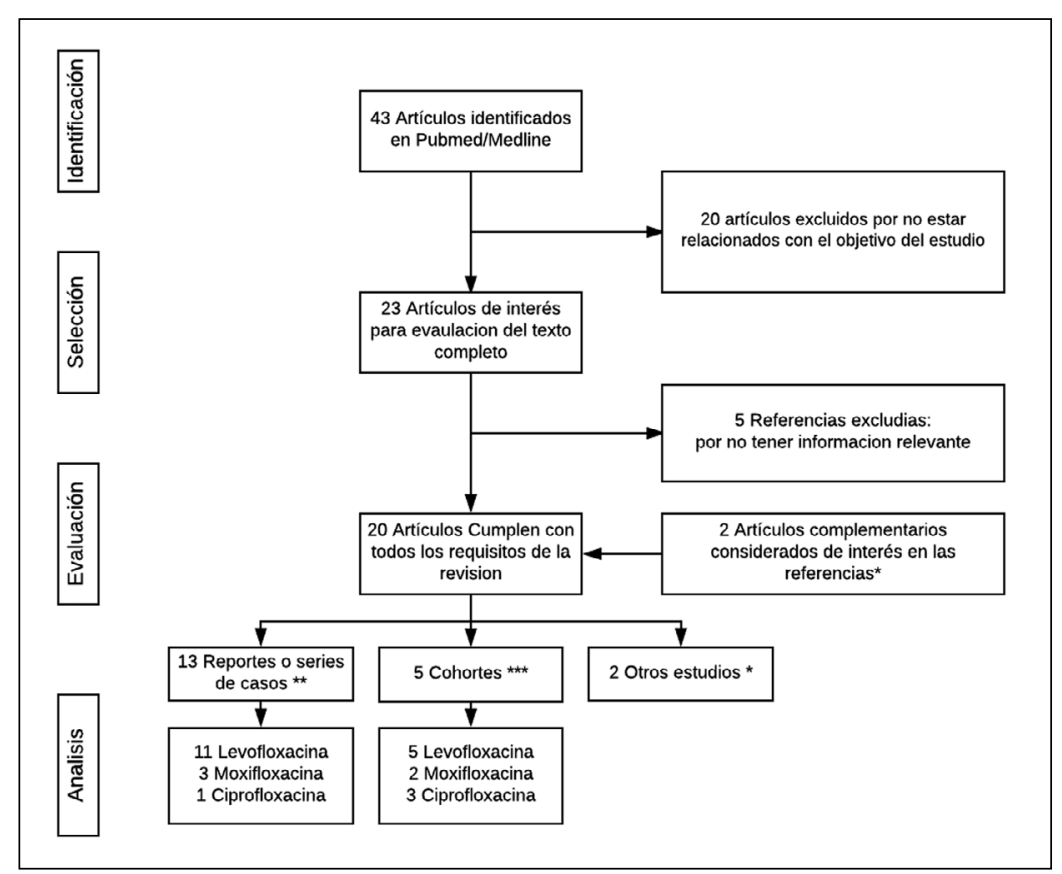

Figura 1. Flujograma de búsqueda. *Artículos que evalúan el posible mecanismo hipoglucemiante de las quinolonas. ${ }^{* *} 2$ artículos presentaron 2 reportes de caso cada uno. ***En las cohortes se evaluó más de una quinolona.

Tabla 1. Clasificación de la relevancia clínica de la disglucemia asociada a las quinolonas (interacciones medicamento enfermedad de mecanismo farmacodinámico) ${ }^{5}$

\begin{tabular}{|llll|}
\hline Fluoroquinolona & $\begin{array}{l}\text { Evento } \\
\text { disglucemico }\end{array}$ & Gravedad/probabilidad (referencias) & $\begin{array}{l}\text { Relevancia clínica: } \\
\text { Nivel de riesgo }\end{array}$ \\
\hline Levofloxacina & Hipoglucemia & Grave/Probable $(6-8,10,11,14-23)$ & 1: Muy alto \\
Moxifloxacina & Hipoglucemia & Grave/Probable $(9,19,21,23)$ & 1: Muy alto \\
Ciprofloxacina & Hipoglucemia & Grave/Probable $(13,19,20,21)$ & 1: Muy alto \\
Levofloxacina & Hiperglucemia & Leve/Probable $(19-23)$ & 3: Riesgo medio \\
Moxifloxacina & Hiperglucemia & Leve/Probable $(23)$ & 3: Riesgo medio \\
Ciprofloxacina & Hiperglucemia & Leve/Probable $(19,20,21)$ & 3: Riesgo medio \\
\hline
\end{tabular}

Dentro del grupo de antibióticos los macrólidos se les considera que tienen un bajo efecto sobre la homeostasis de la glucosa, por tanto los estudios de cohorte comparan las incidencias de hipoglucemia e hiperglicemia de las quinolonas con estos antibióticos, El análisis comparativo de incidencias y riesgos relativos (RR) de hipoglucemias, atribuidas al tratamiento con fluoroquinolonas versus al asociado a los macrólidos (considerados como no hipoglucemiantes) mostró que la utilización de fluoroquinolonas aumenta el riesgo de hipoglucemia, la Figura 2 muestra los valores de $\mathrm{RR}$ obtenidos en cada una de las cohortes para cada quinolona.
En 3 cohortes analizadas la incidencia de disglucemias, principalmente hipoglucemia, fue definida como grave al ser causa de ingreso al departamento de urgencias o causa de hospitalización, en pacientes en tratamiento conjunto de antidiabéticos con quinolonas hasta 30 días después de la prescripción de las antimicrobianos, las otras 2 cohortes evaluaron pacientes hospitalizados en tratamiento con quinolonas y tomaron como valores de hipoglucemia por debajo de 50 y $70 \mathrm{mg} / \mathrm{dl}$ y valores superiores a $200 \mathrm{mg} / \mathrm{dl}$ como hiperglucemia, sin clasificar la severidad de los eventos. (Las características de estos estudios analíticos se pueden observar en la Tabla 3). 


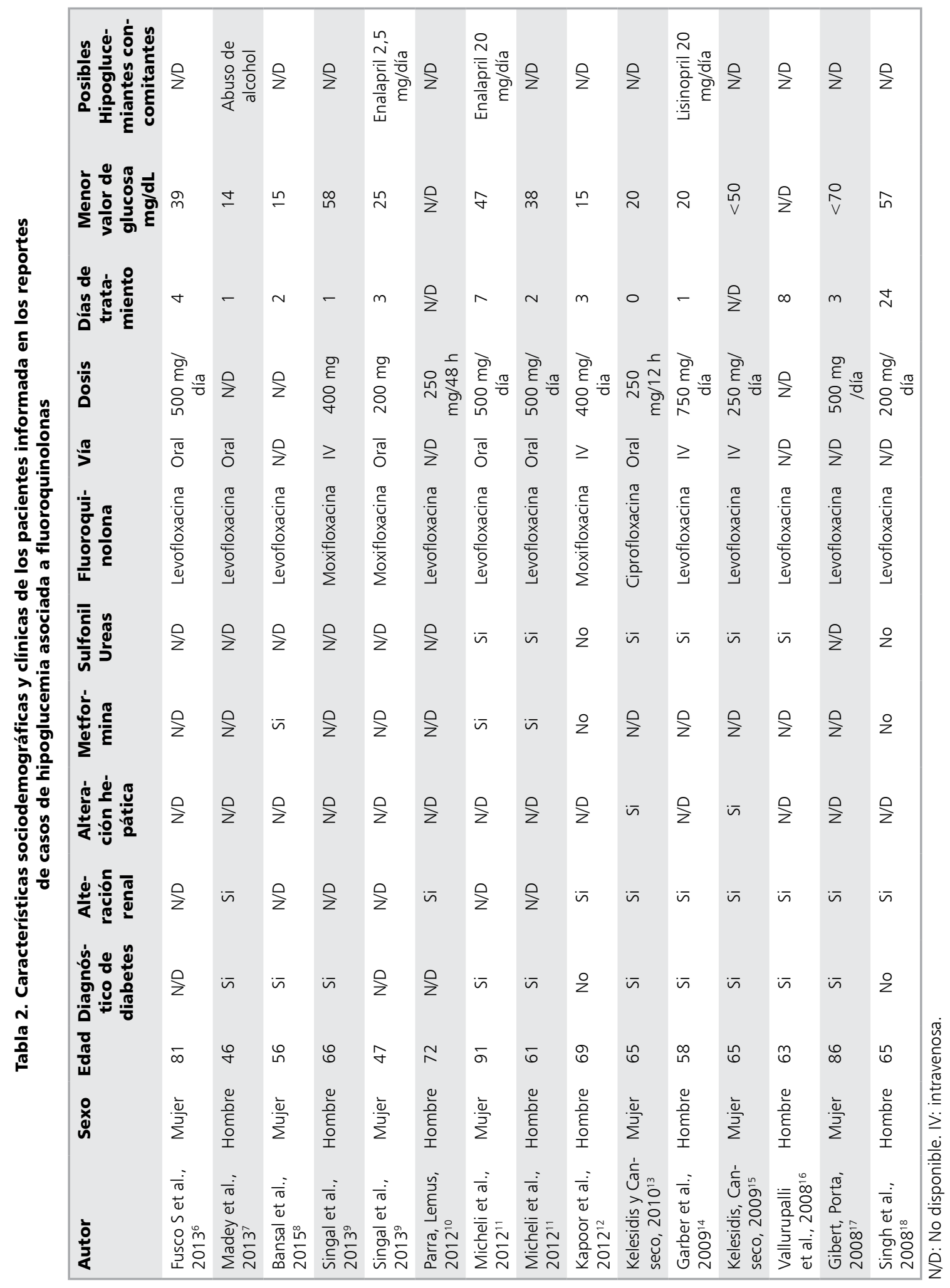


Disglucemia y fluoroquinolonas - J. Granados et al

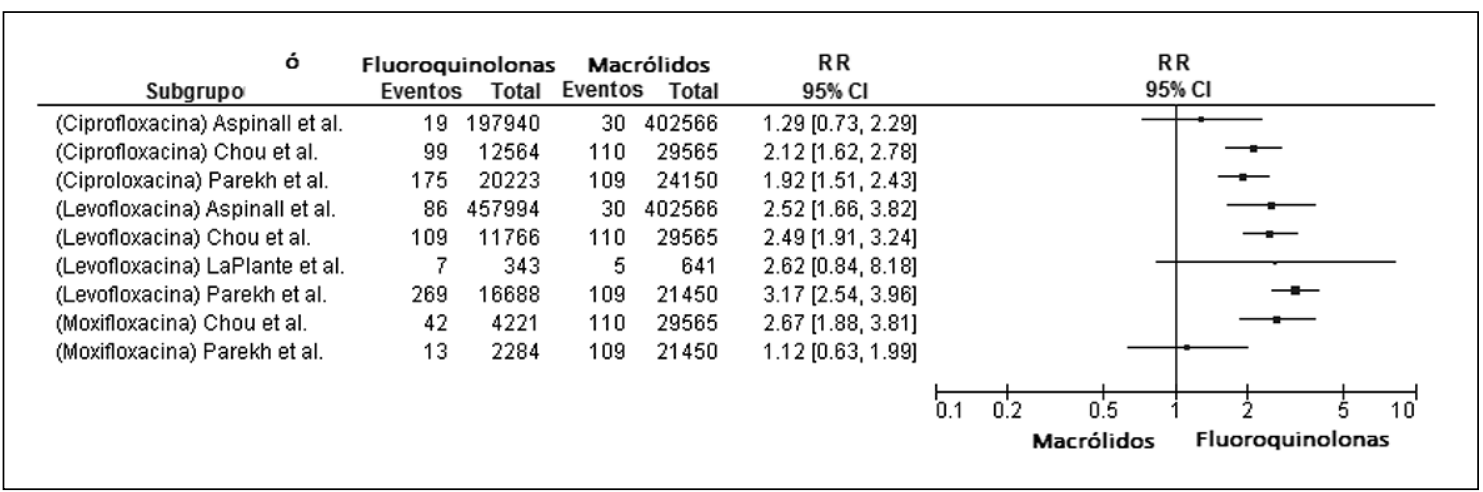

Figura 2. Comparación de incidencias y riesgos relativos (RR) de hipoglucemia entre fluoroquinolonas y macrólidos.

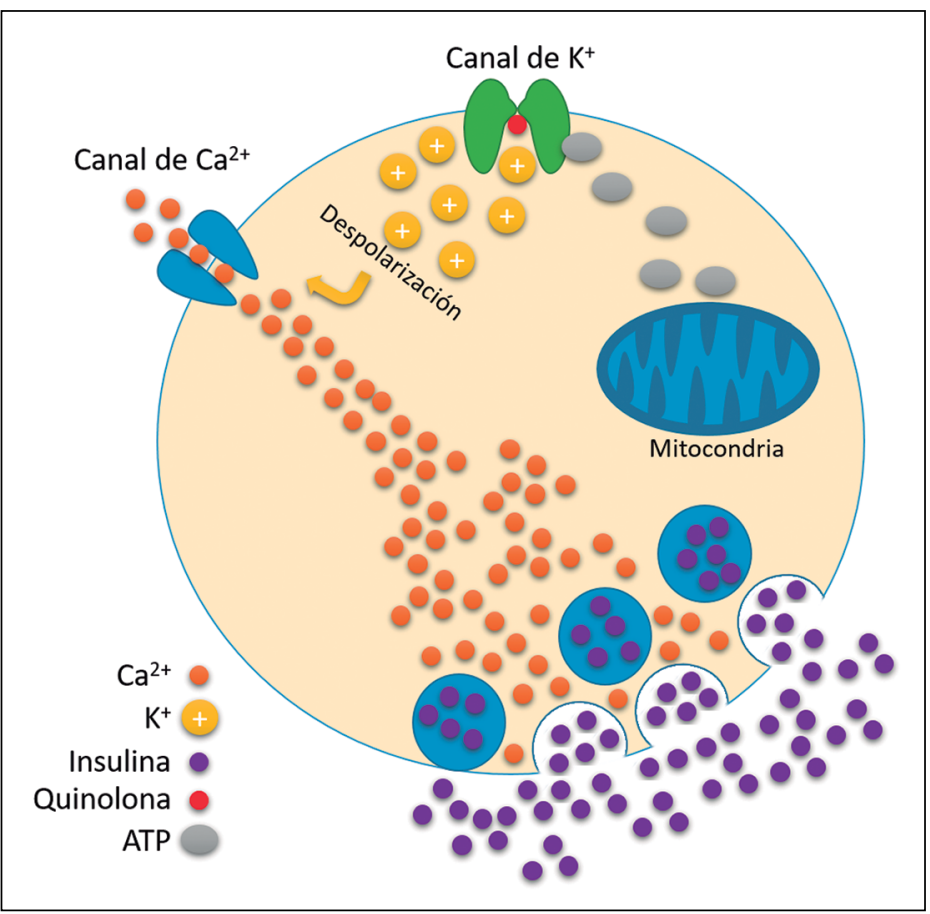

Figura 3. Posible mecanismo del efecto hipoglucemiante de las fluoroquinolonas 24,25 .
Dentro de las principales limitaciones encontradas en estas cohortes se manifestó la dificultad para captar los pacientes ya que los datos fueron tomados de forma retrospectivas y no siempre se encontraron todos los exámenes de laboratorio, adicionalmente, aunque las cohortes presentaron grandes tamaños de muestra, los casos aún son bajos debido a la baja frecuencia de aparición de hipoglucemia producida por quinolonas.

\section{Discusión}

Los resultados de la presente revisión sustentan la afirmación de que las fluoroquinolonas pueden causar cambios en la homeostasis de la glucosa $^{3}$, esencialmente hipoglucemia (Tablas 1 y 2). En este sentido, es importante destacar que en pacientes con diabetes el orden decreciente de riesgo del efecto fue levofloxacina $>$ moxifloxaci- 
Tabla 3. Características sociodemográficas y clínicas de los pacientes de las cohortes relacionadas con disglucemia asociada a fluoroquinolonas

\begin{tabular}{|c|c|c|c|c|c|c|c|c|c|c|c|}
\hline \multirow[t]{2}{*}{ Autor } & \multirow{2}{*}{$\begin{array}{c}\text { Sexo } \\
(\% \\
\text { hombres) }\end{array}$} & \multirow{2}{*}{$\begin{array}{l}\text { Edad, } \\
\text { Media } \\
\text { (DE) }\end{array}$} & \multirow{2}{*}{$\begin{array}{l}\text { Diagnós- } \\
\text { tico de } \\
\text { diabetes } \\
(\%)\end{array}$} & \multirow{2}{*}{$\begin{array}{l}\text { Altera- } \\
\text { ción } \\
\text { renal } \\
(\%)\end{array}$} & \multirow{2}{*}{$\begin{array}{c}\text { Alte- } \\
\text { ración } \\
\text { hepática }\end{array}$} & \multirow{2}{*}{$\begin{array}{c}\text { Met- } \\
\text { formina }\end{array}$} & \multirow{2}{*}{$\begin{array}{c}\text { Sulfoni- } \\
\text { lureas } \\
(\%)\end{array}$} & \multirow{2}{*}{$\begin{array}{l}\text { Fluoro- } \\
\text { quinolona }\end{array}$} & \multirow[t]{2}{*}{$\mathbf{n}$} & \multicolumn{2}{|c|}{$\%$ de pacientes } \\
\hline & & & & & & & & & & $\begin{array}{c}\text { hiper- } \\
\text { glucemia }\end{array}$ & $\begin{array}{c}\text { hipo- } \\
\text { glucemia }\end{array}$ \\
\hline \multirow{3}{*}{$\begin{array}{l}\text { Chou } \\
\text { et al., } \\
2013^{24}\end{array}$} & 57,1 & $\begin{array}{c}67,6 \\
(13,0)\end{array}$ & 100 & 10 & 11,6 & 15,7 & 19,0 & Moxifloxacina & 4.221 & 0,69 & 1,00 \\
\hline & 48,4 & $\begin{array}{c}67,0 \\
(12,8)\end{array}$ & 100 & 10,5 & 11,8 & 15,5 & 19,3 & Levofloxacina & 11.766 & 0,39 & 0,93 \\
\hline & 42,2 & $\begin{array}{c}66,4 \\
(13,2)\end{array}$ & 100 & 10,7 & 11,1 & 15,1 & 17,8 & Ciprofloxacina & 12.564 & 0,40 & 0,79 \\
\hline \multirow{2}{*}{$\begin{array}{l}\text { Aspinall } \\
\text { et al., } \\
2009^{25}\end{array}$} & 94,2 & $\begin{array}{c}63,5 \\
(13,5)\end{array}$ & 26,7 & 3,6 & 1 & N/D & N/D & Levofloxacina & 457.994 & 0,02 & 0,02 \\
\hline & 93,7 & $\begin{array}{c}62,8 \\
(13,6)\end{array}$ & 26,3 & 3,1 & 1,3 & N/D & N/D & Ciprofloxacina & 197.940 & 0,01 & 0,01 \\
\hline \multirow{3}{*}{$\begin{array}{l}\text { Parekh et } \\
\text { al., } 2014^{26}\end{array}$} & N/D & N/D & 100 & $\mathrm{~N} / \mathrm{D}$ & $\mathrm{N} / \mathrm{D}$ & N/D & 100 & Levofloxacina & 16.688 & N/D & 1,61 \\
\hline & N/D & N/D & 100 & $\mathrm{~N} / \mathrm{D}$ & N/D & N/D & 100 & Ciprofloxacina & 20.223 & $\mathrm{~N} / \mathrm{D}$ & 0,87 \\
\hline & N/D & $\mathrm{N} / \mathrm{D}$ & 100 & $\mathrm{~N} / \mathrm{D}$ & $\mathrm{N} / \mathrm{D}$ & N/D & 100 & Moxifloxacina & 2.284 & N/D & 0,57 \\
\hline $\begin{array}{l}\text { LaPlante et } \\
\text { al., } 2008^{27}\end{array}$ & 97,4 & $\begin{array}{c}68,0 \\
(12,8)\end{array}$ & 33,8 & N/D & $\mathrm{N} / \mathrm{D}$ & 13.1 & 14.6 & Levofloxacina & 343 & 0,58 & 2,04 \\
\hline $\begin{array}{l}\text { Lodise et } \\
\text { al., } 2007^{28}\end{array}$ & 96,8 & $\begin{array}{l}78,5 \\
(6,5)\end{array}$ & 18,8 & N/D & N/D & N/D & N/D & Levofloxacina & 405 & 7,90 & 1,98 \\
\hline
\end{tabular}

N/D: no disponible.

Tabla 4. Estudios incluidos en la revisión

\begin{tabular}{|lll|}
\hline Autor & Tipo de estudio & Año \\
\hline Fusco S et al ${ }^{11}$ & Reporte de caso & 2013 \\
\hline Madey et al ${ }^{12}$ & Reporte de caso & 2013 \\
\hline Bansal et al ${ }^{13}$ & Reporte de caso & 2015 \\
\hline Singal et al ${ }^{14}$ & Reporte de caso & 2013 \\
\hline Parra y Lemus ${ }^{15}$ & Reporte de caso & 2012 \\
\hline Micheli et al ${ }^{16}$ & Reporte de caso & 2012 \\
\hline Kapoor et al ${ }^{17}$ & Reporte de caso & 2012 \\
\hline Kelesidis y Canseco ${ }^{18}$ & Reporte de caso & 2010 \\
\hline Garber et al ${ }^{19}$ & Reporte de caso & 2009 \\
\hline Kelesidis, Canseco ${ }^{20}$ & Reporte de caso & 2009 \\
\hline Vallurupalli et al ${ }^{21}$ & Reporte de caso & 2008 \\
\hline Gibert, Porta ${ }^{22}$ & Reporte de caso & 2008 \\
\hline Singh et al ${ }^{23}$ & Reporte de caso & 2008 \\
\hline Chou et al ${ }^{24}$ & Cohorte & 2013 \\
\hline Aspinall et al ${ }^{25}$ & Cohorte & 2009 \\
\hline Parekh et al ${ }^{26}$ & Cohorte & 2014 \\
\hline LaPlante et al $^{27}$ & Cohorte & 2008 \\
\hline Lodise et al ${ }^{28}$ & Cohorte & 2007 \\
\hline Saraya A et al & PK/PD & 2004 \\
\hline Ambrose PG et al & Estudio en células animales & 2003 \\
\hline PK/PD: Framacocinetico/farnmacodinámico & \\
\hline
\end{tabular}

PK/PD: Framacocinético/farnmacodinámico. na $>$ ciprofloxacina. Por su parte, sin diferenciar por la presencia de diabetes (pacientes con o sin diabetes -población general-) el riesgo fue mayor con moxifloxacina seguida de ciprofloxacina y levofloxacina, respectivamente.

Relacionado con la seguridad del tratamiento antibiótico con quinolonas en pacientes con diabetes, los resultados de la presente revisión soportan un aumento en la incidencia de eventos hipoglucemiantes mediante interacción farmacodinámica de relevancia 1 (riesgo muy alto) y de hiperglucemias a través de interacción farmacodinámica de relevancia 3 (riesgo medio). Según los estudios de cohorte identificados, en población con o sin diabetes (población general), la moxifloxacina es la fluoroquinolona con mayor incidencia de efecto hipoglucemiante, seguida de ciprofloxacina y levofloxacina, respectivamente. Sin embargo, en pacientes con diabetes, la levofloxacina fue la fluoroquinolona con mayor probabilidad de causar el evento de hipoglucemia, seguida de la moxifloxacina y ciprofloxacina, respectivamente. Por tanto, en pacientes con diabetes, la ciprofloxacina es la que tiene menor probabilidad de generar hipoglucemia. Esta información debería ser considerada especialmente en los pacientes con 
diabetes ambulatorios, ya que estos son los que podrían tener limitaciones para un seguimiento y valoración del efecto en la glucemia asociado al inicio y terminación del tratamiento con este tipo de antibióticos.

La edad avanzada parece ser un factor que aumenta el riesgo de presentarse disglucemias asociadas a fluoroquinolonas. En este sentido, en los reportes de caso y los estudios de cohorte, la edad promedio se ubicó entre los 66 y 78 años. Adicionalmente, la alteración renal y el uso concomitante de sulfonil-ureas se asociaron a una mayor incidencia de eventos de hipoglucemia.

\section{Mecanismo hipoglucemiante}

Las quinolonas se unen a los canales de potasio dependientes de ATP sobre la membrana celular de las células pancreáticas $\beta$, esto conlleva a una inhibición de la salida de iones de potasio del interior de la célula pancreática así como su acumulación, causando que el potencial de membrana se vuelva más positivo. Esta despolarización permite la apertura de canales de calcio voltaje dependientes que a su vez incrementan la fusión de los gránulos transportadores de insulina con la membrana celular aumentado la liberación de insulina a circulación sistémica. Si bien el bloqueo en los canales de potasio dependientes de ATP es necesario para ejercerse el efecto de liberación de insulina, este mecanismo no parece ser suficiente para explicar la hipoglucemia causada por las quinolonas $^{8}$ (Figura 3).

\section{Mecanismo hiperglucemiante}

Los estudios en animales han demostrado una disminución relacionada con la dosis en la liberación de insulina de las células en respuesta a un desafío de glucosa después de 7 días de exposición a fluoroquinolonas? .

\section{Orden de la actividad hipoglucemia de las quinolonas}

En pacientes con diabetes y factores de riesgo para presentar hipoglucemias, que requieran un tratamiento con una fluoroquinolona teniendo presente el tipo de infección y perfil de sensibilidad, se debería considerar el posible orden de probabilidad de presentar hipoglucemia asociado al tratamiento con estos fármacos (levofloxacina $>$ moxifloxacina $>$ ciprofloxacina). Esta información podría ser de interés, en el momento de elegir una fluoroquinolona en este tipo de pacientes, en especial de edad avanzada y ambulatorios, los cuales carecen de un monitoreo más estricto del efecto de la prescripción de dichos antibióticos en la glucemia.

\section{Limitaciones}

La principal limitación del presente estudio se debe a que la búsqueda se limitó a la base de datos PubMed/Medline. Sin embargo, este efecto pudo disminuirse con la inclusión de las referencias bibliográficas de los artículos consideradas de interés para el tema.

\section{Conclusiones}

Las fluoroquinolonas pueden causar disglucemia, esencialmente hipoglucemia en pacientes con o sin diabetes (población general) con un riesgo en orden decreciente, moxifloxacina $>$ ciprofloxacina $>$ levofloxacina. Por su parte, en pacientes con diabetes el riesgo obedece al siguiente orden: levofloxacina $>$ moxifloxacina $>$ ciprofloxacina. Aunque la incidencia de esta condición sea baja, en pacientes en tratamiento antidiabético, con factores de riesgo, esencialmente edad avanzada y enfermedad renal, existe mayor probabilidad de presentarse hipoglucemias graves, interacción farmacodinámica clínicamente relevante (riesgo muy alto) que debería ser tenida en cuenta en el momento de la prescripción de estos fármacos.

\section{Referencias}

1. Association AD. Standards of Medical Care in Diabetes-2016 Abridged for Primary Care Providers. Clin Diabetes 2016; 34 (1): 3-21.

2. Yun J-S, Ko S-H. Risk Factors and Adverse Outcomes of Severe Hypoglycemia in Type 2 Diabetes Mellitus. Diabetes Metab J 2016; 40 (6): 423-32.

3. May M, Schindler C. Clinically and pharmacologically relevant interactions of antidiabetic drugs. Ther Adv Endocrinol Metab 2016; 7 (2): 69-83.

4. Luna B, Feinglos MN. Drug-induced hyperglycemia. JAMA 2001; 286 (16): 1945-8.

5. Hooper DC, Jacoby GA. Mechanisms of drug resistance: quinolone resistance. Ann N Y Acad Sci 2015; 1354 (1): 12-31. 
6. Korbel L, Spencer JD. Diabetes Mellitus and Infection: An Evaluation of Hospital Utilization and Management Costs in the United States. J Diabetes Complications 2015; 29 (2): 192-5.

7. Ghaly H, Kriete C, Sahin S, Pflöger A, Holzgrabe U, Zünkler BJ, et al. The insulinotropic effect of fluoroquinolones. Biochem Pharmacol 2009; 77 (6): 1040-52.

8. Saraya A, Yokokura M, Gonoi T, Seino S. Effects of fluoroquinolones on insulin secretion and beta-cell ATP-sensitive K+ channels. Eur J Pharmacol 2004; 497 (1): 111-7.

9. Ambrose PG, Bhavnani SM, Cirincione BB, Piedmonte M, Grasela TH. Gatifloxacin and the elderly: pharmacokinetic-pharmacodynamic rationale for a potential age-related dose reduction. J Antimicrob Chemother 2003; 52 (3): 435-40.

10. Amariles P, Andrés Giraldo N, Faus J M. Interacciones medicamentosas: aproximación para establecer y evaluar su relevancia clínica. Med Clínica 2007; 129 (1): 27-35.

11. Fusco S, Reitano F, Gambadoro N, Previti M, Russo G, Basile G, et al. Severe hypoglycemia associated with levofloxacin in a healthy older woman. J Am Geriatr Soc 2013; 61 (9): 1637-8.

12. Madey JJ, Hannah JA, Lazaridis C. Central pontine myelinolysis following acute hypoglycemia. Clin Neurol Neurosurg 2013; 115 (10): 2299-300.

13. Bansal N, Manocha D, Madhira B. Life-threatening metabolic coma caused by levofloxacin. Am J Ther 2015; 22 (2): e48-51.

14. Singal DK, Mittal A, Prakash A. Recurrent episodes of hypoglycemia induced by moxifloxacin. Indian J Pharmacol 2013; 45 (3): 301-2.

15. Parra-Riffo H, Lemus-Peñaloza J. Hipoglicemia severa por levofloxacino: caso clínico y revisión de la literatura. Nefrol Madr 2012; 32 (4): 546-7.

16. Micheli L, Sbrilli M, Nencini C. Severe hypoglycemia associated with levofloxacin in Type 2 diabetic patients receiving polytherapy: two case reports. Int J Clin Pharmacol Ther 2012; 50 (4): 302-6.

17. Kapoor R, Blum D, Batra A, Varma N, Lakshmi K, Basak $\mathrm{P}$, et al. Life-threatening hypoglycemia with moxifloxacin in a dialysis patient. J Clin Pharmacol 2012; 52 (2): 269-71.
18. Kelesidis T, Canseco E. Quinolone-induced Hypoglycemia: A Life-threatening but Potentially Reversible Side Effect. Am J Med 2010; 123 (2): e5-6.

19. Garber SM, Pound MW, Miller SM. Hypoglycemia associated with the use of levofloxacin. Am J Health-Syst Pharm AJHP Off J Am Soc Health-Syst Pharm 2009; 66 (11): 1014-9.

20. Kelesidis T, Canseco E. Levofloxacin-induced Hypoglycemia: A Rare but Life-threatening Side Effect of a Widely Used Antibiotic. Am J Med 2009; 122 (3): e3-4.

21. Vallurupalli S, Huesmann G, Gregory J, Jakoby MG. Levofloxacin-associated hypoglycaemia complicated by pontine myelinolysis and quadriplegia. Diabet Med J Br Diabet Assoc 2008; 25 (7): 856-9.

22. Gibert AE, Porta FS. Hypoglycemia and levofloxacin: a case report. Clin Infect Dis Off Publ Infect Dis Soc Am 2008; 46 (7): 1126-7.

23. Singh M, Jacob JJ, Kapoor R, Abraham J. Fatal hypoglycemia with levofloxacin use in an elderly patient in the post-operative period. Langenbecks Arch Surg Dtsch Ges Für Chir 2008; 393 (2): 235-8.

24. Chou H-W, Wang J-L, Chang C-H, Lee J-J, Shau W-Y, Lai M-S. Risk of severe dysglycemia among diabetic patients receiving levofloxacin, ciprofloxacin, or moxifloxacin in Taiwan. Clin Infect Dis Off Publ Infect Dis Soc Am 2013; 57 (7): 971-80.

25. Aspinall SL, Good CB, Jiang R, McCarren M, Dong D, Cunningham FE. Severe dysglycemia with the fluoroquinolones: a class effect? Clin Infect Dis Off Publ Infect Dis Soc Am 2009; 49 (3): 402-8.

26. Parekh TM, Raji M, Lin Y-L, Tan A, Kuo Y-F, Goodwin JS. Hypoglycemia After Antimicrobial Drug Prescription for Older Patients Using Sulfonylureas. JAMA Intern Med 2014; 174 (10): 1605-12.

27. LaPlante KL, Mersfelder TL, Ward KE, Quilliam BJ. Prevalence of and risk factors for dysglycemia in patients receiving gatifloxacin and levofloxacin in an outpatient setting. Pharmacotherapy 2008; 28 (1): 82-9.

28. Lodise T, Graves J, Miller C, Mohr JF, Lomaestro B, Smith RP. Effects of gatifloxacin and levofloxacin on rates of hypoglycemia and hyperglycemia among elderly hospitalized patients. Pharmacotherapy 2007; 27 (11): 1498-505. 